\title{
Expanding clinical genetics services in a rural state in the post-genomic, technology-connected age: A dispatch from Mississippi
}

\author{
Brian Kirmse* \\ Department of Pediatrics, University of Mississippi Medical Center, Division of Medical Genetics, \\ Jackson, MS, USA
}

\begin{abstract}
Like many rural states and developing countries, Mississippi lacks sufficient clinical genetics services to meet the needs and demands of patients with rare disorders in the post-genomic age. The essential responsibilities of the Medical Geneticist and Genetic Counselor are to establish a genetic diagnosis in a patient with a suspected genetically-determined disorder, to provide evidence-based genetic counseling and to directly provide or link the patient to treatment and other necessary resources. In Mississippi we have used both telemedicine (asynchronous and synchronous) and other alternative delivery arrangements to augment statewide capacity. Here we provide a narrative of our experience and a potentially generalizable model for other health systems with a clinical genetics service shortfall.
\end{abstract}

Keywords: Clinical genetics, clinical genomics, telemedicine, telehealth, advanced practice nurses, nurse practitioner, community health worker

"To understand the world, you must first understand a place like Mississippi"

William Faulkner

\section{Introduction}

Mississippi is unique for many good reasons. To start, it's the birthplace of Eudora Welty, Robert Johnson, Elvis and the Muppets. It is not unique in that, like many states and areas in the country right now, it struggles to keep pace with the demand for clinical genetics services engendered by the recent and ongoing explosion of genomic testing and targeted treatments. It is generally accepted as fact by those of us who practice traditional Clinical Genetics medicine, that there are not enough providers, that the providers are clustered in large urban areas, and that as a subspecialty we are woefully bad at recruiting people to the profession [1]. These observations certainly hold true in Mississippi where, as of 2017, there were 2 Clinical Geneticists (just 1 FTE) and 3 Genetic Counselors for 4 million people, all practicing in the capital of Jackson where there was no active residency program (yet) or genetic counseling program (yet) to feed the current and future clinical needs.

\footnotetext{
${ }^{*}$ Corresponding author: Brian Kirmse, MD Department of Pediatrics, University of Mississippi Medical Center, Division of Medical Genetics, Jackson, MS, USA. E-mail: bkirmse@umc.edu.
} 
The purpose of this article is not, however, to complain about or directly address the clinical genetics workforce shortage or even to call for more students and trainees in our field, both of which have been addressed elsewhere [2,3]. Those are tasks better left to our national colleges and societies. The purpose of this article is to first of all distill the essential and specialized roles of clinical genetics service providers in the post-genomic era and then describe the ways in which we in Mississippi have leveraged our state and institutional strengths in non-traditional ways to expand access to clinical genetic services here. Many of these alternative approaches have been employed out of necessity - too many patients in need, too few geneticists and counselors. Our hope is that hospitals, health systems and states that find themselves with similar challenges (and we think there are many) might benefit from our experience.

\section{What's supposed to happen in the genetics clinic?}

The answer is probably straightforward enough: those of us in the genetics clinic care for patients with known or suspected genetic disorders. In reality, though, it is more complicated than that. Generally, those of us practicing Clinical Genetics know the myriad roles we play in a health system even if we don't have the time to enumerate them to our generalist or subspecialist colleagues and we have become quite adept at switching hats - our first appointment of the day is a toddler with Down syndrome followed by a visit with middle-aged woman with a family history of early breast cancer which is interrupted by an emergent call from the NICU about an encephalopathic neonate with an ammonia of 2000. From the outside it is sometimes difficult to figure out what a Clinical Geneticist does. I suppose there are days when I have trouble pinning it down, too.

It's easier to figure out what a cardiologist does - nearly everyone knows what a heart is and at least some of the ways a heart can go wrong in disease. So, if the heart is the lesion locus for a cardiologist (and the brain for a neurologist, the kidney for a nephrologist, etc.), then perhaps the most expedient way to focus our practice, at least from the outside gaze, is to say that a clinical geneticist is one who helps patients with suspected or confirmed DNA lesions (I'm not the first one to suggest this construct and I think it was Victor McKusick who wrote in somewhere, but I can't readily put my hands on the reference).

Through another lens we are physicians who help diagnose and care for patients with rare disorders. We are natural sleuths and enjoy finding our culprit and pinning a rap on him. It so happens that a majority of rare disorders are genetically determined. In the past, even just 10 years ago, rare disease medicine was relegated to diagnosing and treating those disorders that had an easily picked out clinical or biochemical phenotype (a form pleine), something that could be measured or had validated diagnostic criteria to be met. Nowadays rare disorders, when looked at from the perspective of the interrupted gene and the era of clinically available exome/genome sequencing, take on an impressive multidimensionality as they heterogeneously sit upon a spectrum of clinical severity and an array of pleiotropy.

But the essential job of a genetics clinic and consult service is to evaluate a patient with a suspected genetic disorder, formulate and execute a genetic testing strategy and then, when a genetic lesion is located, counsel the patient and family about the implications of that lesion including any available treatment and appropriate social/medical resources [Fig. 1]. So, in concept the genetics clinic has a simple job, even as the practical realities of its work become more complex and the group of providers who have chosen to fuel it dwindle in number.

\section{Augmenting capacity in the clinic}

Increasingly, it is the responsibility of the genetics clinic to establish a genetic diagnosis (find a bone fide genetic lesion) in patients who are suspected of having a genetically-determined disorder. It has 


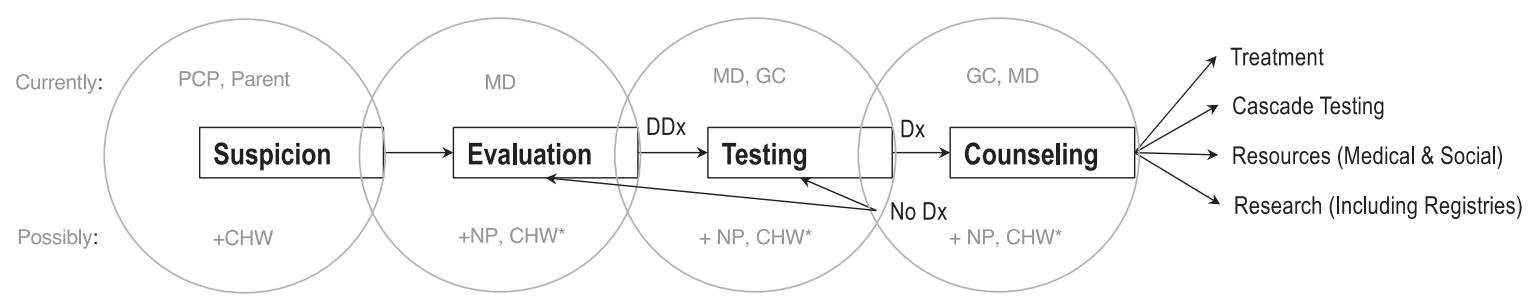

Fig. 1. Workflow in the Clinical Genetics Clinic. PCP, Primary Care Provider; CHW, Community Health Worker; MD, Medical Doctor; NP, Nurse Practitioner; GC, Genetic Counselor; Dx, Diagnosis; DDx, Differential Diagnosis; *Provider-supporting roles and/or narrow, supervised scope of practice in areas where there are no genetic services.

always been our lot, but no longer can we hide behind clinical diagnostic rates of 10-15\% [4] and vague "gestalt" clinical feelings about a patient's diagnosis. Now that clinical genetic testing is more ubiquitous and has firmly entered the public consciousness with the popularity of ancestry informative genetic tests, and "genetic health screens" like 23 andMe, the volume of patients and referring providers seeking genetic tests is increasing. Indeed, in the post-genomic era in which genomic testing (large gene panels, whole exome sequencing (WES), etc.) is readily available in the clinic and costing less each year and in which a "new" genetically-determined disorder is discovered almost weekly, genetic diagnostic rates for unique (even common) phenotypes is at an historic high. Recent studies of WES report diagnostic rates as high as 50\% [4] (and possible more on top of that with whole genome sequencing and clinical transcriptomics) one could argue that it is a primary duty to ensure that the $10 \%$ of the population thought to be affected by a rare disorder be, when possible, told what it is that ails them.

\subsection{Welcome the clinical genetics nurse practitioner}

Traditionally the general genetics clinic work force at academic/tertiary care medical centers in the US has revolved around the MD Geneticist - Genetic Counselor dyad. The first step in the evaluation process (after suspicion of a genetic disorder is established which we come back to later) has been to gather history (both family and medical) and phenotype the patient. In the MD-GC dyads that I have worked in it was usually the GC's role to gather information and the MD's role to evaluate that information and examine/phenotype. In addition to being cumbersome and sometimes redundant, this relationship usually falls apart after a few years since most GCs do not get their Master's in Genetic Counseling to (only) be an information gatherer or scribe to a physician in perpetuity. In my experience, GCs like to counsel patients and so in our model of genetic care delivery we have made a conscious effort to uncouple the dyad and allow GCs to counsel. While the GCs concentrate on counseling this might leave information gathering to the physicians alone and while geneticists are able enough to conduct an entire evaluation, it results in the ability to see fewer patients. We've found that some of the time-consuming and straightforward aspects of a clinical genetics practice - from evaluation to testing and treatment - can be successfully delegated to another type of healthcare professionals in our midst, namely the advanced practice nurse.

While the supply of MD Geneticists and GCs is not keeping pace with current or projected needs, schools that educate advanced practice nurses (nurse practitioners, NPs) have been graduating an increasing number of students. The American Association of Colleges of Nursing reports $8200 \mathrm{NP}$ graduates in 2008 and just 10 years later in 2017 there were 28,000. Just for oblique comparison's sake, in 2019 Abacar et al. reported 400 GCs graduated each year. Further anecdotal proof of this discrepancy between the workforce and the need is illustrated in the fact that when we, in Mississippi, advertised for an NP to work on our team, we had 22 qualified applicants in the first week after the 
position was posted. In contrast, we have had no applicants for the GC position that we opened up almost 2 years ago. As a field, we would be crazy not to capitalize on this trove of potential talent.

Many of us who practice metabolic genetics have become comfortable with the model of NPs as newborn screening coordinators, but we have found that NPs fit in nicely in many roles in the genetics clinic, including but certainly not limited to, information gathering and algorithm-based evaluations for narrow indications like abnormal newborn screens, suspected connective tissue disease or autism/intellectual disability We have also found roles for NPs in health supervision and care coordination for children and adults with chromosomal disorders as well as maintaining infusion programs. I've heard many of the arguments against having NPs as integral parts of the clinical genetic team but in reality, when part of a multidisciplinary care team that includes (an) MD Geneticist(s) and GCs, NPs excel and provide much needed specialized care to patients with known or suspected genetic disorders. Of course, for the model to be successful, there is a requisite investment of time for training and education. To date it has been up to individual genetics teams/divisions to train NPs and provide foundational clinical genetics training since there are no formal postgraduate NP genetics training programs or certificates. There is evidence that others in the field are thinking this way [5]. In September 2019, the Medical College of Wisconsin held its first (of many, one hopes) Clinical Genetics Advanced Practice Provider Conference in Milwaukee, possibly heralding a future workforce boon to the genetic clinics in the US. Thirty-five genetics NPs attended the conference and came from 19 different states. All were employed in diverse roles in the genetic clinic and hospital service - from inpatient consults, to subspecialty clinics (neurology, cardiology), to general genetics clinics seeing anything from connective tissue disorders to intellectual disability.

In our experience training an NP in genetics and inculcating him or her into the rarefied world of genetic disease and its peculiar nuances and sensitivities can take about as much time as it would to train a new resident-about a year or two. This process for us is in part didactic (lectures, case discussions, journal clubs, etc.) and experiential. Experience comes from an accumulation of exposure to clinical scenarios while working with a physician and GC. Just like a resident, this investment begins with a closer working relationship between the physician and NP but gradually evolves into more independence (never complete independence in our model) as the needs of the clinic/service and the strengths, interests and skills of the NP emerge and mature. Moreover, we have cultivated a nonpunitive, open, team-based approach to patient care so no provider, including the MDs, feels that he or she can't learn from or lean on any other member of the team. Practically speaking this culture is achieved through having clinics in the same suite on the same days (we take up lots of rooms), a weekly clinical case conference wherein we discuss cases and review the literature for pertinent disorders. We also hold a weekly all-hands "triage meetings" the aim of which is to review referrals to ensure they get to the right provider/clinic. These triage meetings are also a great opportunity for the team to review differential diagnoses and "red flag" presentations for common referral indications.

Currently our team employs two NPs: one is assigned to newborn screening, metabolic clinic and the small ERT infusion program we have. The second is assigned to general, non-metabolic inpatient consults (which are seen with an MD geneticist via asynchronous telehealth model), autism/ID evaluations and care coordination/health supervision for patients with known chromosomal disorders.

\subsection{Synchronous telehealth from Jackson to the rest of the state}

Technology (telehealth, mobile medicine and even artificial intelligence) continues to change the way healthcare is delivered [6] and the expectations of patients. Google has already drastically altered the rare disease diagnosis landscape: who among us hasn't had a patient come to them recently for an initial evaluation with a list of signs and symptoms in one hand and an internet generated differential diagnosis in the other? And is right. 
Traditional telehealth, which in our state has been used to increase access to subspecialty care in rural communities, is usually accomplished using fancy technology (expensive Polycom or Cisco 2way synchronous video carts with the appropriate scopes (stetho-, oto-, ophtalmo-) attached on the patient end) and between traditional healthcare delivery site (hospitals, clinics). This can work well providing the technology is reliable, you have institutional support (resources and personnel to maintain the technology) and there is a telehealth savvy staff member at the distant site to troubleshoot when things go (technologically) wrong. The younger generations, who were weaned on flat screen devices and indeed cannot conceive of living life without them as an adjunct brain, will demand that more and more of their care - at least straightforward transactional care (like, for example, the ordering of genetic testing for a "family history of") - be delivered to them wherever they are. We have to be prepared to accommodate their clinical genetics needs (all of it: evaluation, testing, counseling) lest they begin to rely on less-than-ideal alternatives (read Google and/or self-enthroned "experts" they meet on the Internet).

Our University's Center for Telehealth is one of two HRSA-designated Centers of Telehealth Excellence in the country and we have used its resources and ever-expanding network of statewide "spoke" sites to expand access to genetic services in Mississippi. We've done this in 2 ways, one traditional and one novel. Over the last 3 years our Children's Hospital has established remote pediatric multidisciplinary clinics all over the state and at each of these clinics there is at least one exam room that is equipped with a Cisco-based videoconferencing cart that is connected to our offices and clinic in Jackson as well as anywhere else we happen to be thanks to connectivity through our mobile devices (using Jabber).

We began our telegenetics program with genetic counseling appointments in Tupelo and Biloxi from Jackson but quickly, because of demand and need, expanded to two other sites (Hattiesburg and Meridian) and to include initial evaluations and follow-up visits. Again, the lack of providers (MDs and GCs) in Jackson limited the amount of time we could spend in telegenetics clinics around the state so to solve this problem we hired a remote MD (to evaluate) and remote GC (to counsel) patients in all the outlying clinics leaving the Jackson providers to concentrate on patients coming in-person to Jackson.

\subsection{Asynchronous telegenetics in the NICU}

The clinical genetics needs in the NICU run the gamut, but the most important function of the genetics team, at least initially, is to establish a genetic diagnosis in a sick child with birth defects or another dramatic neonatal presentation. Metabolic and neurological syndromes aside, many of these NICU evaluations revolve around children with congenital anomalies and/or dysmorphia and the role of the Geneticist, after gathering information about the family, pregnancy and birth of course, is to examine the patient for physical clues that point towards a unifying diagnosis. Typically, further clinical phenotyping (imaging, biochemical labs, etc.) and genetic testing (chromosomal analysis, gene testing, exome/genome) is recommended either to confirm a clinical diagnosis or to refine a differential diagnosis.

We have recently begun using an asynchronous (store-and-forward) telehealth model in our NICU for non-urgent/emergent cases. The model employs a Genetics NP who is in Jackson and responsible for most of the initial contact, contracting and consenting with the family, for the clinical information gathering and for obtaining a preset series of high definition photos. A remote clinical geneticist/dysmorphologist then reviews the history, the chart, the photos and renders a clinical opinion and genetic testing strategy through direct communication with the team and family as well as through documentation of a consult note in the electronic medical record (EMR). During the 2 years in which we have been using this model we have noted a non-inferior diagnostic rate ( $21 \%$ of consults 
before panel or exome testing) to those reported in the literature [7] and have maintained high levels of satisfaction among referring providers and patient families.

\section{Counselors should be counseling (and educating)}

Up-to-date, culturally sensitive and evidence-based genetic counseling has become essential before and after a diagnosis of a genetic disorder. Genetic counseling is a process that is usually not finished in a single visit since the implications of any given genetic diagnosis may change over time (as new understandings of the disorder and treatments emerge) and because a patient and his or her family's risks and needs may also change over time. Good counseling takes time and preparation; good counseling is a high-touch endeavor [8].

As previously mentioned, we have dispensed with the traditional MD-GC dyad in our practice in service of a model of care delivery that is more optimized to provider strength and scope, the hope being that by allowing providers, in this case GCs, to do what they are trained to do and like to do most, they will actually enjoy what they are doing and skirt the burn-out that many healthcare providers in many sectors are experiencing [9]. If we agree that there is a genetic services workforce problem in this country, then we need to do everything to ensure the genetics service providers we do have on the front lines of patient care want to stay there. Generally speaking, counselors should be counseling, not scribing, faxing, scheduling, hunting down records, triaging calls for physicians or many of the other dissipating tasks that can eat up a day and deplete job satisfaction. Of course, none of these tasks are below a counselor or physician to do, but, again, good counseling takes time and any time recouped from administrative tasks is more time in front of patients, helping.

A practical and frustrating bottleneck for scaling genetic counseling services in our state lies in the fact that GCs are not licensed as professionals so that in the clinic, if counseling services are to be billed for, a physician needs to be involved in the case even if it's to briefly meet a patient and/or family before and after a counseling session to highlight the main points of the visit and to answer any outstanding questions. Even though physicians here typically see other patients at the same time (usually for evaluations or follow up visits), and even though we have "broken the dyad", this practical consideration still requires that the physician and counselor be in clinic at the same time, a situation that, in a busy academic medical center that demands GC and physician effort outside the clinic, is not easily achieved all day, every day. We have addressed this limitation in two ways. Firstly, we have used technology - specifically Cisco Jabber, Doxyme, etc. - to connect patient and GC in clinic with a physician who is working in his/her office, a different clinic or in the adjacent hospital. Secondly, we have taken advantage of the evolving organ system-defined niches in genetics disorders (like neurogenetics, cardiogenetics and pediatric cancer genetics) and allowed our GCs who "live" in our Division to periodically embed in other divisions and collaborate with non-genetics physicians in order to facilitate appropriate genetic testing and post-test counseling for patients with a more narrow spectrum of genetic disease (i.e., channelopathies or cardiomyopathies in the Cardiology clinic, epilepsy in the neurology clinic).

Recruiting genetic counselors to a rural state is difficult. Mississippi does not have its own GC Masters Program yet and this does not help matters. While not a guarantee of future workforce stability, an in-state GC program helps by juxtaposing potential "first jobs" with an already vetted group of rising GCs. Until we have a GC training program, our Division, with the indefatigable support from our Department of Pediatrics, has maintained two Genetic Counseling Assistant (GCA) positions. GCAs are typically employed to help with the aforementioned administrative tasks that take physicians and GCs out of the clinic and while this is true for the GCA positions in Mississippi (we have a very strict "it's not scut if it's for a patient" policy), we have gone further. We have also limited the tenure of a GCA 
to no more than 2 years and have hired only those post-baccalaureates who are intent on becoming a GC, genetics research scientist or even an MD, provided that person is interested in becoming a Clinical Geneticist or at least a genetic savvy physician who knows the difference between a microarray and exome. The GCA position here in Mississippi has become something of an internship and incubator for those who will go out and pursue a clinical genetics-oriented career and might one day make their way back to Mississippi to practice. Indeed, one of our team's GCs was once a GCA here and our last two GCAs are both currently in GC schools in other states.

Investing in the future of the genetics workforce and nurturing the workforce you have is only one half of the battle, though. Educating the public (potential and future patients/consumers of genomic medicine) in the foundations of genetic and health literacy is the other important component of the genetics provider/patient dialogue. By several metrics our state is considered the unhealthiest in the Union with the highest levels of diabetes, high blood pressure and obesity translating into an almost 4 -year difference in life expectancy compared to the national average $[10,11]$. Unhealthy states tend to be those with low health literacy. Unsurprisingly, Mississippi ranks among the lowest states in health literacy scores $[12,13]$, and while there is no reputable study suggesting unhealthy states like Mississippi shoulder a disproportionate burden of genetic disorders, there is at least some evidence that health literacy correlates with genetic literacy and that a large proportion of the American public may lack "genetic testing awareness" [14]. We should worry that if something isn't done to address this societal knowledge gap at a population level that we may be enabling health disparities in genomic medicine. Our ultimate goal as a field and as a society should not only be to supply the clinical genetics workforce and innovate the care delivery system but also to help ensure that those patients in need of clinical genetic services have the basic tools and knowledge to understand, not only their own genetics-related health risks, but also the concepts and practical options (testing, treatment, etc.) that are foundational to the clinical genetics encounter and what issues therefrom.

To this end, genetics education - at all levels and in a diversity of settings - is a major priority for our Division. Along with teaching the teachers (foundational lectures for medical students in clinical genetics and how genetics/genomics factors into the practice of medicine), members of our division (lead by a GC Genetics Education Director) also teach GC students from outside programs, NP students, nursing students as well as residents, fellows and faculty from across the hospital in Grand Rounds and didactic conferences. We also regularly provide outreach education (lectures, meet and greets) at community pediatrics and obstetrics practices and annual meetings for the state chapters of professional societies. Moreover, our Division's education mission extends to the young public: we regularly attend (and are a hit at) DNA Day at the Mississippi Children's Museum and other science-based community activities. It only remains that when we one day have the time, that we more fully engage with our

State primary and secondary educational systems to ensure genetic literacy is taught early and often to the students cum citizens of Mississippi.

\section{Future directions and challenges}

From where we are in Mississippi, we see nothing but a growing need for genetic services and education in our state. From a workforce standpoint, we will continue to nurture clinical genetics service providers where ever they can be found - we have a newly-accredited Medical Genetics Residency Program and plans of the distant horizon for a Genetic Counseling Master's Program. We will continue to grow up the current and next generation of Clinical Genetics NPs and we will continue to educate and encourage all current and future healthcare providers at our medical center to "think genetically" (as Kurt Hirschhorn was fond of saying) at every level and with every patient encounter. 
From a technology standpoint, I think the future will be increasingly mobile (as in direct patient care over mobile devices) and before long we will be piloting "at home" follow up visits for our patients with straightforward metabolic genetics conditions like MCAD (Medium chain Acyl Co-A dehydrogenase deficiency), galactosemia and biotinidase deficiency, saving them from long round trips in the care. For our Phenylketonuria (PKU) patients we are planning a remote patient monitoring and education program aimed at keeping adolescents and adults on diet and adhering to their medications as well as a Project ECHO-type engagement [15] with primary care physicians in the rural reaches of our state taking care of PKU patients who find it hard to get to Jackson for their visits.

Right now, access to genetic services is not equal across the US, especially in Mississippi. The richer and more educated you are the more likely you are to get appropriate genetics care [16]. Barriers are myriad and unequally distributed in society. And if this is true in the US, it is also true in more resource limited settings throughout the world. According to the Global Commission to End the Diagnostic Odyssey for Children with Rare Disorders, there is an average of 1 MD Geneticist per 222,000 people in Western developed countries compared to 1 per 1.28 million and 4.5 million people in Latin America and South Africa, respectively, highlighting the fact that access inequality to clinical genetics services is even greater in parts of the world where there wealth and basic healthcare delivery disparities are larger. It is difficult to conceive of any imminent geopolitical shift that will impact this reality any time soon and result in a higher number of trained MD Geneticists and Genetic Counselors in developing countries, but it's possible to imagine non-traditional remedies that might help address the gap. Relatively inexpensive awareness and genetics education programs for primary care providers and other types of healthcare providers as well as alternative delivery arrangements for high-impact genetic services (basic prenatal counseling and testing, pediatric diagnostic evaluations for common indications, identification of families whose pedigrees that are suspicious for hereditary cancer syndrome, to name a few) might alleviate the service shortfall in some situations. Community Healthcare Workers (CHW), who receive education and training for a very narrow clinical indication and by definition do not have a tertiary healthcare-related education, have been successfully deployed in many places throughout the world - both rural and urban - and for myriad public health problems (from management of maternal to child transmission HIV to identification and treatment of pediatric pneumonia) [17, 18]. According to the World Health Organization (WHO), which has recognized CHWs as important part of the global health care delivery system for 50 years, "community health workers should be members of the communities where they work, should be selected by the communities, should be answerable to the communities for their activities, should be supported by the health system but not necessarily a part of its organization, and have shorter training than professional workers." [19] For any community motivated to improve and expand genetic services, even in underserved communities in "developed" countries (in Mississippi, perhaps), CHWs might represent a potential boon and an untapped resource for those with genetic disorders.

The very real obstacles to equitable access that stem from social issues like poverty, race and history are wicked and compound all of the issues of workforce and care delivery that have been discussed above. I don't think these are necessarily insurmountable problems or reasons for abject despair. The solutions, as always, will be found in working together and in using what we have right in front of us.

\section{Acknowledgments}

The author would like to acknowledge all the members of the Division of Medical Genetics in the Department of Pediatrics at UMMC who work hard every day to provide world-class care for patients in Mississippi. 


\section{References}

[1] M. Summar, Save the biochemical geneticists! Mol Genet Metab 113 (2014), 2-3.

[2] J.M. Hoskovec, R.L. Bennett, M.E. Carey, J.E. DaVanzo, M. Dougherty, S.E. Hahn, B.S. LeRoy, S. O’Neal, J.G. Richardson and C.A. Wicklund, Projecting the supply and demand for certified genetic counselors: A workforce study, J Genet Couns 27 (2018), 16-20.

[3] M. Penon-Portmann, J. Chang, M. Cheng and J.T. Shieh, Genetics workforce: distribution of genetics services and challenges to health care in California, Genet Med 22(1) (2020), 227-231.

[4] Y. Yang, D.M. Muzny, F. Xia, Z. Niu, R. Person, Y. Ding, P. Ward, A. Braxton, M. Wang, C. Buhay, et al. Molecular findings among patients referred for clinical whole-exome sequencing, JAMA 312 (2014), 1870-1879.

[5] M. Campion, C. Goldgar, R.J. Hopkin, C.A. Prows and S. Dasgupta, Genomic education for the next generation of health-care providers. Genet Med 21(11) (2019), 2422-2430.

[6] E.R. Dorsey and E.J. Topol, State of telehealth, N Engl J Med 375 (2016), 1400.

[7] D.T. Miller, M.P. Adam, S. Aradhya, L.G. Biesecker, A.R. Brothman, N.P. Carter, D.M. Church, J.A. Crolla, E.E. Eichler, C.J. Epstein, et al. Consensus statement: chromosomal microarray is a first-tier clinical diagnostic test for individuals with developmental disabilities or congenital anomalies, Am J Hum Genet 86 (2010), 749-764.

[8] B. Heald, L. Rybicki, D. Clements, J. Marquard, J. Mester, R. Noss, M. Nardini, J. Polk, B. Psensky, C. Rigelsky, et al. Assessment of clinical workload for general and specialty genetic counsellors at an academic medical center: A tool for evaluating genetic counselling practices, NPJ Genom Med 1 (2016), 16010.

[9] A.A. Wright and I.T. Katz, Beyond burnout - redesigning care to restore meaning and sanity for physicians, $N$ Engl $J$ Med 378 (2018), 309-311.

[10] U.S.B.O.D. Collaborators, A.H. Mokdad, K. Ballestros, M. Echko, S. Glenn, H.E. Olsen, E. Mullany, A. Lee, A.R. Khan, A. Ahmadi, et al. The State of US Health, 1990-2016: Burden of Diseases, Injuries, and Risk Factors Among US States. JAMA 319 (2018), 1444-1472.

[11] C. Global Burden of Cardiovascular Diseases, G.A. Roth, C.O. Johnson, K.H. Abate, F. Abd-Allah, M. Ahmed, K. Alam, T. Alam, N. Alvis-Guzman, H. Ansari, et al. The Burden of Cardiovascular Diseases Among US States, 1990-2016, JAMA Cardiol 3 (2018), 375-389.

[12] S.C. Bailey, G. Fang, I.E. Annis, R. O'Conor, M.K. Paasche-Orlow and M.S. Wolf, Health literacy and 30-day hospital readmission after acute myocardial infarction, BMJ Open 5 (2015), e006975.

[13] I.O. Medicine, Health Literacy: A Prescription to End Confusion. In Health Literacy: A Prescription to End Confusion, L. Nielsen-Bohlman, A.M. Panzer, andD.A. Kindig, eds. (Washington (DC) (2004).

[14] M. Krakow, C.L. Ratcliff, B.W. Hesse and A.J. Greenberg-Worisek, Assessing genetic literacy awareness and knowledge gaps in the US population: Results from the health information national trends survey, Public Health Genomics 20 (2017), 343-348.

[15] S. Arora, K. Thornton, G. Murata, P. Deming, S. Kalishman, D. Dion, B. Parish, T. Burke, W. Pak, J. Dunkelberg, et al. Outcomes of treatment for hepatitis C virus infection by primary care providers, N Engl J Med 364 (2011), $2199-2207$.

[16] C. Nikolaidis, D. Duquette, K.E. Mendelsohn-Victor, B. Anderson, G. Copeland, K.J. Milliron, S.D. Merajver, N.K. Janz, L.L. Northouse, S.A. Duffy, et al. Disparities in genetic services utilization in a random sample of young breast cancer survivors, Genet Med 21 (2019), 1363-1370.

[17] A. Ciapponi, S. Lewin, C.A. Herrera, N. Opiyo, T. Pantoja, E. Paulsen, G. Rada, C.S. Wiysonge, G. Bastias, L. Dudley, et al. Delivery arrangements for health systems in low-income countries: An overview of systematic reviews, Cochrane Database Syst Rev 9 (2017), CD011083.

[18] B. Wahl, S. Lehtimaki, S. Germann and N. Schwalbe, Expanding the use of community health workers in urban settings: a potential strategy for progress towards universal health coverage, Health Policy Plan 35(1) (2020), 91-101.

[19] L.Z. Bhutta ZA, G. Pariyo and L. Huicho, Global experience of community health workers for delivery of health related Millennium Development Goals: A systematic review, country case studies, and recommendations for integration into national health systems. In. (WHO/Global Health Workforce Alliance), (2010), pp. 1-391. 